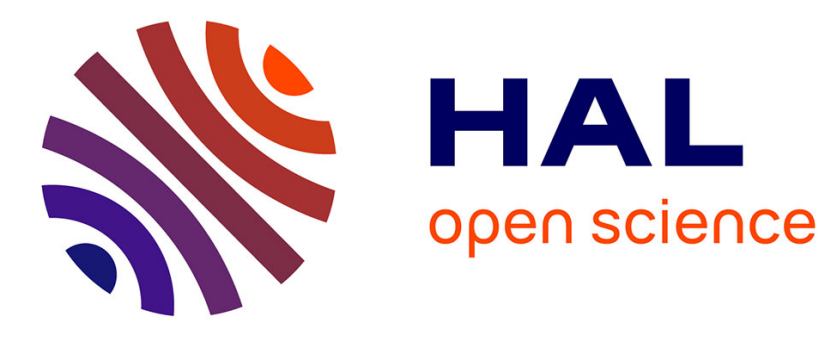

\title{
Photochemical preparation of silver nanoparticles supported on zeolite crystals
}

Moussa Zaarour, Mohamad El Roz, Biao Dong, Richard Retoux, Roy Aad, Julien Cardin, Christian Dufour, Fabrice Gourbilleau, Jean-Pierre Gilson, Svetlana Mintova

\section{To cite this version:}

Moussa Zaarour, Mohamad El Roz, Biao Dong, Richard Retoux, Roy Aad, et al.. Photochemical preparation of silver nanoparticles supported on zeolite crystals. Langmuir, 2014, 30 (21), pp.62506256. 10.1021/la5006743. hal-01138057

\section{HAL Id: hal-01138057 https://hal.science/hal-01138057}

Submitted on 4 Apr 2017

HAL is a multi-disciplinary open access archive for the deposit and dissemination of scientific research documents, whether they are published or not. The documents may come from teaching and research institutions in France or abroad, or from public or private research centers.
L'archive ouverte pluridisciplinaire HAL, est destinée au dépôt et à la diffusion de documents scientifiques de niveau recherche, publiés ou non, émanant des établissements d'enseignement et de recherche français ou étrangers, des laboratoires publics ou privés. 


\title{
Photochemical Preparation of Silver Nanoparticles Supported on Zeolite Crystals
}

\author{
Moussa Zaarour, ${ }^{\dagger}$ Mohamad El Roz, ${ }^{\dagger}$ Biao Dong, ${ }^{\dagger}$ Richard Retoux ${ }^{\ddagger}{ }^{\ddagger}$ Roy Aad, ${ }^{\S}$ Julien Cardin, ${ }^{\S}$ \\ Christian Dufour, ${ }^{\S}$ Fabrice Gourbilleau, ${ }^{\S}$ Jean-Pierre Gilson, ${ }^{\dagger}$ and Svetlana Mintova*, ${ }^{\dagger}$ \\ ${ }^{\dagger}$ LCS, ${ }^{\ddagger}$ CRISMAT, and ${ }^{\S}$ CIMAP, ENSICAEN, Université de Caen, CNRS, 6 bd du Maréchal Juin, 14050 Caen, France
}

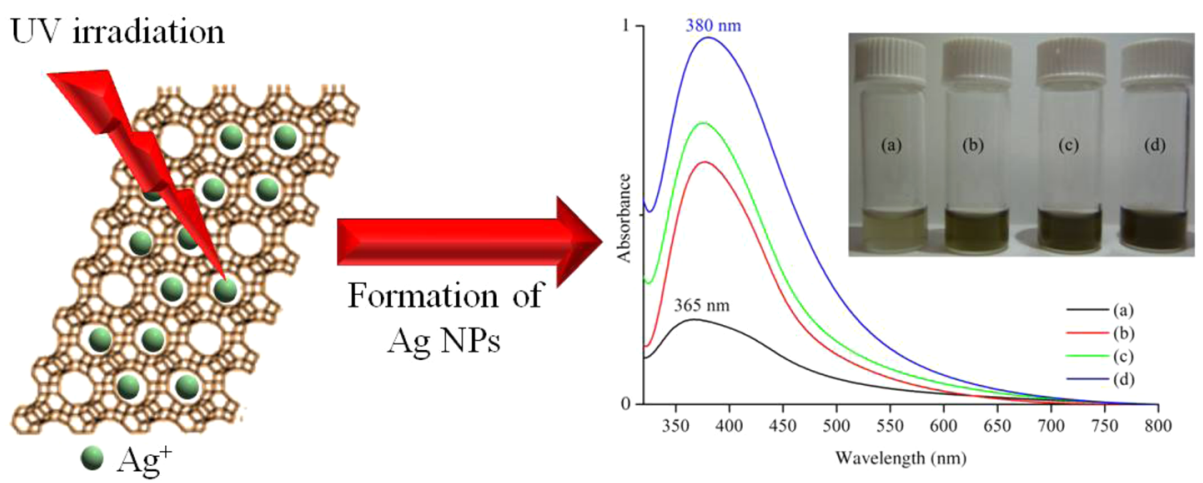

\begin{abstract}
A facile and rapid photochemical method for preparing supported silver nanoparticles (Ag-NPs) in a suspension of faujasite type (FAU) zeolite nanocrystals is described. Silver cations are introduced by ion exchange into the zeolite and subsequently irradiated with a $\mathrm{Xe}-\mathrm{Hg}$ lamp $(200 \mathrm{~W})$ in the presence of a photoactive reducing agent (2-hydroxy-2methylpropiophenone). UV-vis characterization indicates that irradiation time and intensity $\left(I_{0}\right)$ influence significantly the amount of silver cations reduced. The full reduction of silver cations takes place after $60 \mathrm{~s}$ of a polychromatic irradiation, and a plasmon band of Ag-NPs appears at $380 \mathrm{~nm}$. Transmission electron microscopy combined with theoretical calculation of the plasmon absorbance band using Mie theory shows that the Ag-NPs, stabilized in the micropores and on the external surface of the FAU zeolite nanocrystals, have an almost spheroidal shape with diameters of 0.75 and $1.12 \mathrm{~nm}$, respectively. Ag-NPs, with a homogeneous distribution of size and morphology, embedded in a suspension of FAU zeolites are very stable ( $\sim 8$ months), even without stabilizers or capping agents.
\end{abstract}

\section{INTRODUCTION}

Silver nanoparticles are currently the focus of intensive research because of their catalytic, antibacterial, and optical properties. ${ }^{1}$ Their interesting optical properties, more precisely, their plasmonic properties, make them highly desirable in various applications like sensors, ${ }^{2}$ OLEDs, ${ }^{3}$ and photovoltaic solar cells. $^{4-6}$ Their plasmonic properties depend strongly on their size, morphology, and density. ${ }^{7}$ Therefore, a systematic modification of these parameters allows the tuning of their plasmonic response over the whole visible spectrum.

In solution, silver nanoparticles agglomerate to form clusters or even large particles and hence lose their optical properties. To prevent this and maintain the plasmonic properties, Ag-NPs are usually charged or dispersed in media such as noble gases, organic scaffolds, or a porous matrix (zeolites). ${ }^{7}$

Zeolites are crystalline aluminosilicates with a framework made of $\mathrm{SiO}_{4}$ and $\mathrm{AlO}_{4}$ tetrahedra, in which the negatively charged $\mathrm{Al}$ sites are neutralized by the charge-balancing extraframework cations, such as $\mathrm{K}^{+}, \mathrm{Li}^{+}, \mathrm{Na}^{+}$, etc. ${ }^{8}$ These charge compensating cations are noncovalently bonded to the zeolite and offer the possibility of replacing them with organic molecules or metals with interesting properties for optical applications. $^{9-14}$ In fact, the use of zeolites for optical applications has already attracted the attention of researchers because of their diverse structures, high porosity, high thermal stability, and availability in different sizes and morphologies. ${ }^{10}$ The incorporation of zeolites into an optical device offers the possibility of stabilizing and organizing the photoactive guests as well as preventing intermolecular interactions that reduce or even quench their photophysical properties. ${ }^{11}$ As a result, several studies reported the use of zeolites as suitable supports for Ag-NPs. ${ }^{12-14}$

Ag-NPs are generally prepared by either thermal reduction, ${ }^{15}$ microwave reduction, ${ }^{16}$ sonochemical reduction, ${ }^{17}$ chemical reduction, ${ }^{18}$ or photoreduction of silver cations $\left(\mathrm{Ag}^{+}\right)$in solution. ${ }^{19-21}$ Thermal treatment is a classical way to prepare Ag-NPs: the solution containing silver cations is heated in the presence of a reducing agent such as $\mathrm{H}_{2}$ or $\mathrm{CO}$, introduced 
directly into the reaction mixture, or generated in situ. ${ }^{15}$ Microwave-assisted reduction presents several advantages like a more homogeneous heating, a shorter reaction time, and an easier nucleation of Ag-NPs. ${ }^{16}$ The sonochemical process is used for the preparation of Ag-NPs when the particle size strongly depends on the type of reducing agent applied. ${ }^{17}$ Using sodium borohydride, a strong reducing agent, results in spherical Ag-NPs $10 \mathrm{~nm}$ in size, while with a weak reducing agent, such as sodium citrate, the formation of Ag-NPs $\sim 3 \mathrm{~nm}$ in size is observed. ${ }^{17}$ Moreover, chemical treatment is an efficient process for preparing Ag-NPs in solution with good control of their size and shape. The key to a rational design is to find the proper combination of silver precursors, a reducing agent, stabilizers, and reaction conditions (rate and $\mathrm{pH}){ }^{18}$ The drawback of these methods is the use of relatively large amounts of reducing agents and their subsequent elimination in an additional step. Furthermore, Ag-NPs are generally sensitive to heat and oxygen; the addition of capping and stabilizing agents is usually recommended to preserve their structure and prevent their aggregation in solution.

The photoassisted synthesis (photoreduction) of silver is another method used for the preparation of Ag-NPs, albeit less practiced. It allows the preparation of stable nanoparticles of Ag-NPs by irradiation of a reaction mixture with a light source (laser or lamp) in the presence of photoreducing agents without the need to introduce stabilizers or surfactants. ${ }^{19-21}$ Their size and the time needed for their preparation are directly proportional to the irradiation power of the light source. For example, with a low-power lamp (4 W), irradiation for $9 \mathrm{~h}$ is needed to produce $19 \mathrm{~nm}$ diameter $\mathrm{Ag}-\mathrm{NPs}^{21}{ }^{21}$ while with a stronger source $(150 \mathrm{~W})$, the reaction takes only $45 \mathrm{~min}$. However, in the latter case, the Ag-NPs are polydisperse and smaller than when they are prepared using a lower-energy lamp. $^{21}$

In this work, we report a facile and rapid preparation of AgNPs, supported on FAU type zeolite crystals, using a photochemical reduction method. The silver cations are introduced into the FAU zeolite nanocrystals $(\mathrm{Si} / \mathrm{Al}=1.2)$ by ion exchange and subsequently reduced (to $\mathrm{Ag}^{0}$ ) by irradiation with a $\mathrm{Xe}-\mathrm{Hg}$ lamp $(200 \mathrm{~W})$ for 5-60 s. The irradiation intensity and time have a direct influence on the $\mathrm{Ag}$ nanoparticles and their plasmonic properties. The microstructure and optical properties of the Ag-NPs are monitored by high-resolution transmission electron microscopy (HRTEM) and UV-vis spectroscopy, respectively.

\section{- EXPERIMENTAL SECTION}

Raw Materials. Silver nitrate $\left(\mathrm{AgNO}_{3}\right)$ was purchased from Alfa Aesar, and 2-hydroxy-2-methylpropiophenone $\left[\mathrm{C}_{6} \mathrm{H}_{5} \mathrm{COC}\right.$ $\left.\left(\mathrm{CH}_{3}\right)_{2} \mathrm{OH}\right]$, benzophenone $\left(\mathrm{C}_{13} \mathrm{H}_{10} \mathrm{O}\right)$, and benzaldehyde $\left(\mathrm{C}_{7} \mathrm{H}_{6} \mathrm{O}\right)$ were purchased from Sigma-Aldrich. Aluminum hydroxide $\left[\mathrm{Al}(\mathrm{OH})_{3}\right.$, Sigma-Aldrich], sodium hydroxide $(\mathrm{NaOH}$, Sigma-Aldrich, $97 \%)$, and colloidal silica $\left(\mathrm{SiO}_{2}\right.$, Ludox-HS 30, 30 wt $\% \mathrm{SiO}_{2}, \mathrm{pH} 9.8$, Aldrich) were used to prepare FAU type zeolite crystals (zeolite $\mathrm{X}$ ) according to the procedure below; these reagents were used without further purification.

Preparation of a FAU Type Zeolite Suspension. The FAU nanocrystals were synthesized from a clear precursor suspension with a 9:0.9:10:200 $\mathrm{Na}_{2} \mathrm{O}: \mathrm{Al}_{2} \mathrm{O}_{3}: \mathrm{SiO}_{2}: \mathrm{H}_{2} \mathrm{O}$ ratio. The suspension was prepared by mixing $\mathrm{Al}(\mathrm{OH})_{3}, \mathrm{NaOH}$, and $\mathrm{SiO}_{2}$ with doubly distilled water and subjected to vigorous stirring at room temperature until a clear suspension was obtained. Then the resulting clear suspension was aged for $24 \mathrm{~h}$ at room temperature prior to the hydrothermal synthesis. The crystallization was performed at $150{ }^{\circ} \mathrm{C}$ for $45 \mathrm{~min}$ in a conventional oven. The zeolite nanocrystals were purified by centrifugation $(25000 \mathrm{rpm}$ for $4 \mathrm{~h}$ ) and dispersed in doubly distilled water $\left(\mathrm{dd} \mathrm{H}_{2} \mathrm{O}\right)$ until the $\mathrm{pH}$ of the decanted suspension reached 7 . Finally, the purified FAU zeolite sample was centrifuged and decanted; the resulting FAU slurry is further ion-exchanged as described below.

Ion Exchange of FAU Zeolites. The FAU slurry $(100 \mathrm{mg})$ was sonicated in a $10 \mathrm{~mL}$ aqueous solution of $\mathrm{AgNO}_{3}(0.1 \mathrm{M})$ for $6 \mathrm{~h}$, and the product was purified by centrifugation and washed three times with doubly distilled water to remove any excess of silver. The ionexchanged FAU zeolite in a water suspension will hereafter be termed FAU- $\mathrm{Ag}^{+}$. The as-prepared suspension was freeze-dried; the color of the $\mathrm{FAU}-\mathrm{Ag}^{+}$sample prior reduction is white. The $\mathrm{FAU}-\mathrm{Ag}^{+}$powder sample was redispersed in water $\left(0.25 \mathrm{~g} / \mathrm{L}\right.$ in $\left.\mathrm{H}_{2} \mathrm{O}\right)$. One milliliter of this FAU-Ag ${ }^{+}$suspension containing $2.64 \times 10^{-4} \mathrm{mg}$ of $\mathrm{Ag}^{+}$was then added to $0.1 \mathrm{~mL}$ of 2-hydroxy-2-methylpropiophenone $(0.096 \mathrm{M}$ in ethanol, 99\%). This mixture was stirred while being irradiated with a $\mathrm{Xe}-\mathrm{Hg}$ polychromatic lamp with an intensity varying from 20 to $100 \%$ for 5-60 s.

Preparation of Ag-NPs in FAU Zeolites. UV irradiation of the FAU- $\mathrm{Ag}^{+}$suspension $(1 \mathrm{~mL})$ was conducted with a polychromatic $\mathrm{Xe}-\mathrm{Hg}$ lamp (LC8-01A spot light, Hamamatsu, L10852, $200 \mathrm{~W}$ ) using a UV light guide (model A10014-50-0110). This device was mounted at the entrance of the reactor containing the zeolite suspensions to establish a "homogeneous" irradiation. The UV irradiation intensity $\left(I_{0}\right)$ at full capacity of the lamp measured with a light power meter (from Hamamatsu) was $55 \mathrm{~mW} / \mathrm{cm}^{2}$ at $366 \mathrm{~nm}$. All samples were stirred in the reactor during the reduction process. The $\mathrm{Xe}-\mathrm{Hg}$ polychromatic lamp intensity varied from 20 to $100 \%$ for $5-$ 60 s. The FAU suspensions containing Ag-NPs will hereafter be termed FAU-Ag.

Characterization of Ag-NPs in FAU Zeolite Suspensions. The UV-vis absorption spectra of the zeolite suspensions containing AgNPs were recorded on a Thermo-electron evolution 500 UV-vis spectrometer working in transmission and in quartz cuvettes with a 1 $\mathrm{cm}$ path length. The zeolite suspension prior to ion exchange was used as a reference for all UV-vis measurements.

Dynamic Light Scattering (DLS) Analysis. The hydrodynamic diameters of the as-prepared suspensions of zeolite nanoparticles and those containing Ag-NPs were determined with a Malvern Zetasizer Nano instrument using a backscattering geometry (scattering angle of $173^{\circ}, \mathrm{He}-\mathrm{Ne}$ laser with a $3 \mathrm{~mW}$ output power at a wavelength of $632.8 \mathrm{~nm}$ ). The DLS analyses were performed on samples with a solid concentration of 1 wt \%.

High-Resolution Transmission Electron Microscopy (HRTEM). The crystal size, morphology, crystallinity, and chemical composition of suspensions of FAU-containing Ag-NPs were characterized using HRTEM coupled with an energy dispersive analysis (EDS) on a 200 $\mathrm{kV}$ JEOL 2010 FEG STEM electron microscope (tilt of $\pm 42^{\circ}$ ) equipped with an EDS (energy dispersive spectrometer, $\mathrm{Si} / \mathrm{Li}$ detector; a double tilt sample holder was used). The suspensions were sonicated for $15 \mathrm{~min}$ prior to their deposition on a holey carbon supported on a nickel grid.

Theoretical Modeling of the Ag Plasmon Band. The simulation of the UV-vis absorbance spectra was performed using the Mie theory. ${ }^{22}$ Simulations were conducted using the refractive index of water taken from the IAPWS ${ }^{23}$ or an adjustable constant refractive index for the surrounding medium. The refractive index of silver was taken from ref 24 . The refractive index of silver was sizecorrected to take into account the reduction of the electron mean free path in nanoparticles. The size correction was conducted by introducing an additional surface contribution into the electronic scattering to retain the Drude part of the dielectric function using the electron scattering rate given by Kriebig: $:^{25}$

$$
\gamma=\gamma_{0}+2 g \frac{\nu_{\mathrm{F}}}{D}
$$

where $\gamma_{0}$ is the bulk scattering rate and $\nu_{\mathrm{F}}$ is the Fermi velocity of silver. The nanoparticle diameter $(D)$ and a phenomenological dimensionless parameter $(g)$ are related to the shape of the particles as well as the material properties and the surrounding dielectric host 
Scheme 1. Preparation of Ag-NPs by Photogeneration of Free Radicals in Suspension


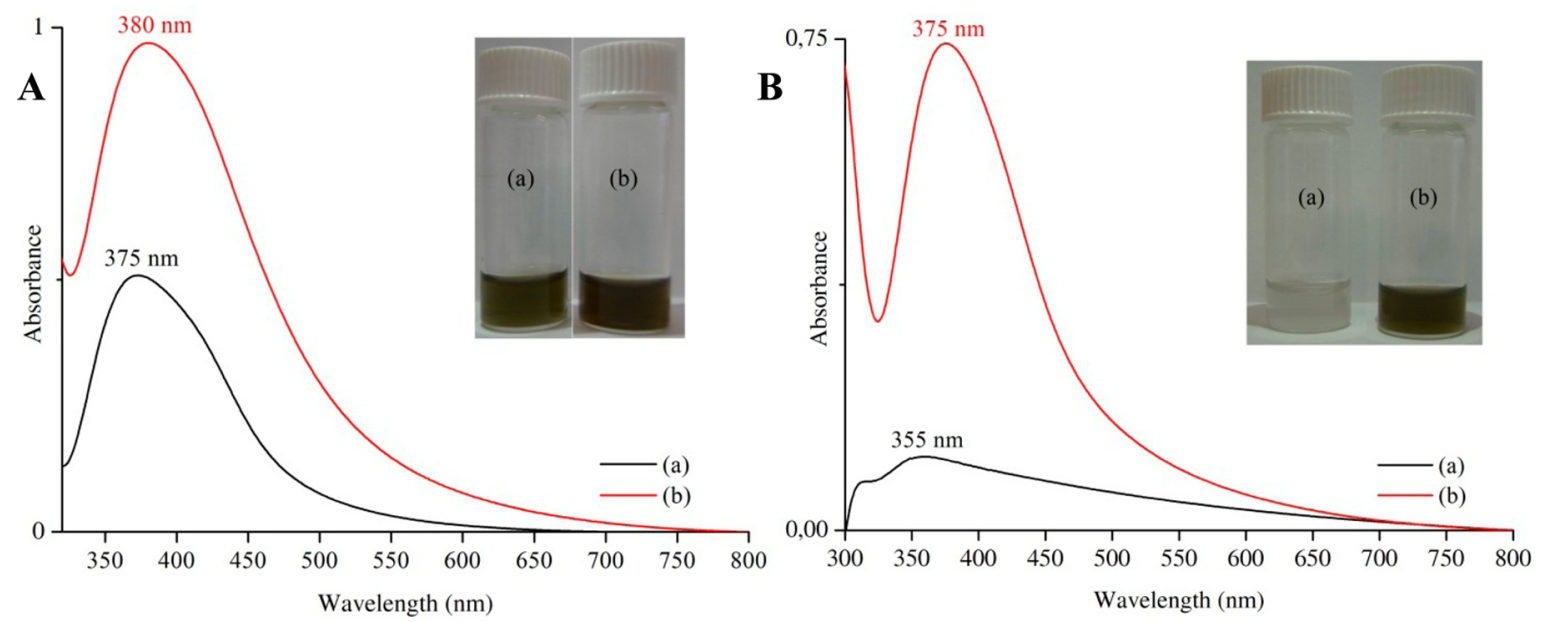

Figure 1. Evolution of the Ag plasmon band in FAU zeolite suspensions after UV treatment $\left(\mathrm{H}_{2} \mathrm{O}\right.$ suspension) (A) at $60 \mathrm{~s}$ at (a) an $I_{0}$ of $20 \%$ or (b) an $I_{0}$ of $100 \%$ and (B) at $15 \mathrm{~s}$ at (a) an $I_{0}$ of $20 \%$ or (b) an $I_{0}$ of $100 \%$.

matrix. For spherical nanoparticles, $g$ is estimated to be $\sim 0.7 \pm 0.1 .^{26}$ The values of $\gamma_{0}$ and $\nu_{\mathrm{F}}$ for silver were taken from ref 27 .

\section{RESULTS AND DISCUSSION}

The photochemical treatment of materials is a fast and efficient method for producing electrons. It requires the use of a photoreducing agent or a photoactive species that becomes electronically excited and rapidly transfers electrons to the $\mathrm{Ag}^{+}$cations (reducing them into $\mathrm{Ag}^{0}$ ), upon irradiation at a specific wavelength. The compatibility between the photoreducing agent and the irradiation source is of prime importance because the catalyst should absorb in the same range as the light excitation to generate the free electrons. Besides the reducing activity, the catalyst is used as a stabilizing agent for the produced species.

In this work, three photoreducing agents, benzophenone, benzaldehyde, and 2-hydroxy-2-methylpropiophenone, are used as photocatalysts. The first two show a very low reactivity in the FAU- $\mathrm{Ag}^{+}$suspension, while the last one is the most compatible with the zeolite suspension and consequently used in all experiments. To ensure the miscibility between the zeolite suspension and the reducing agent, 2-hydroxy-2-methylpropiophenone was first dissolved in ethanol and subsequently added to the FAU- $\mathrm{Ag}^{+}$suspension (in water); the mixture was stirred vigorously during the reaction to ensure homogeneous suspensions free of sedimentation.

The photo-reducing agent used is from the family of hydroxyl-methylphenone, which is well-known to undergo a homolytic $\mathrm{C}-\mathrm{C}$ bond scission ( $\alpha$-cleavage) upon exposure to UV irradiation giving rise to a pair of radicals ${ }^{28}$ (Scheme 1). After the radical formation, the following processes are possible: (1) recombination of radicals going back to the original compound or (2) deactivation of radicals by dissolved oxygen present in the reaction medium (Scheme 1). (3) In the presence of silver cations, the radicals can undergo a redox process giving rise to silver nanoparticles (Ag-NPs) in addition to the cationic form of the radicals that rearrange to form their corresponding ketones. At the end of the reaction, the reducing agent is fully dissociated and will be no longer available to stabilize the produced Ag-NPs.

Influence of Irradiation Intensity on the Formation of Ag-NPs. Because the light source used is of adjustable intensity, the FAU- $\mathrm{Ag}^{+}$suspension is irradiated with different intensities $\left(I_{0}\right)$. At $I_{0}$ values of $<20 \%$, few and nonreproducible reactions are triggered because of a nonstable irradiation at such low intensities. Upon irradiation with an $I_{0}$ of $20 \%$ for $60 \mathrm{~s}$, a plasmon absorbance band at $375 \mathrm{~nm}$ appears in the UV-vis spectrum of the FAU- $\mathrm{Ag}^{+}$suspension and is associated with a green coloration, indicative of the generation of Ag-NPs. Interestingly, a further increase in $I_{0}$ induces a progressive hyperchromic shift of the plasmon absorbance band as well as a slight bathochromic (red) shift. The sample irradiated with an $I_{0}$ of $100 \%$ presents an absorbance twice that at an $I_{0}$ of $20 \%$, and the peak position shifts from 375 to $380 \mathrm{~nm}$ (Figure 1A). This implies that more silver cations are reduced. ${ }^{19}$ The reaction rate is directly proportional to the irradiation intensity 
used. At a low intensity, a small number of radicals are generated, because of the competing deactivation process (Scheme 1); only a few radicals are available for the reduction of silver cations, explaining the low intensity of the plasmon absorbance band. At a high irradiation intensity $\left(I_{0}=100 \%\right)$, many free radicals are generated and are rapidly consumed. This favors the reduction of silver over the deactivation of free radicals and leads to the full reduction of silver cations after only $60 \mathrm{~s}$. This assumption is confirmed by running the reaction for a shorter time (15 s) (Figure 1B). At a low irradiation intensity $\left(I_{0}=20 \%\right)$, a very low intensity band appears at $355 \mathrm{~nm}$ with no change in the color of the suspension. This suggests that the number of free radicals generated is not enough to reduce the silver cations. With an $I_{0}$ of $100 \%$, an intense absorption at $375 \mathrm{~nm}$ is associated with the appearance of a dark green color, indicating that the reaction is almost complete after irradiation for $15 \mathrm{~s}$. For comparison, the $\mathrm{UV}$-visible spectra of the $\mathrm{FAU}-\mathrm{Ag}^{+}$suspension without and with a photoreducing agent (2-hydroxy-2-methylpropiophenone) at time zero are taken and presented in Figure S1 of the Supporting Information. It is clearly seen that after mixing, no absorption band is observed in the spectrum, while the plasmon band appears after $15 \mathrm{~s}$ (Figure 1). These results demonstrate that the photoreducing agent upon exposure to UV irradiation is giving rise to a pair of radicals, and the sequential processes are followed as presented in Scheme 1.

Influence of Irradiation Time on the Formation of AgNPs. The irradiation of a silver-containing zeolite suspension is conducted for different times while fixing the intensity of the light source at $100 \%$. As observed in Figure 2, a plasmon band

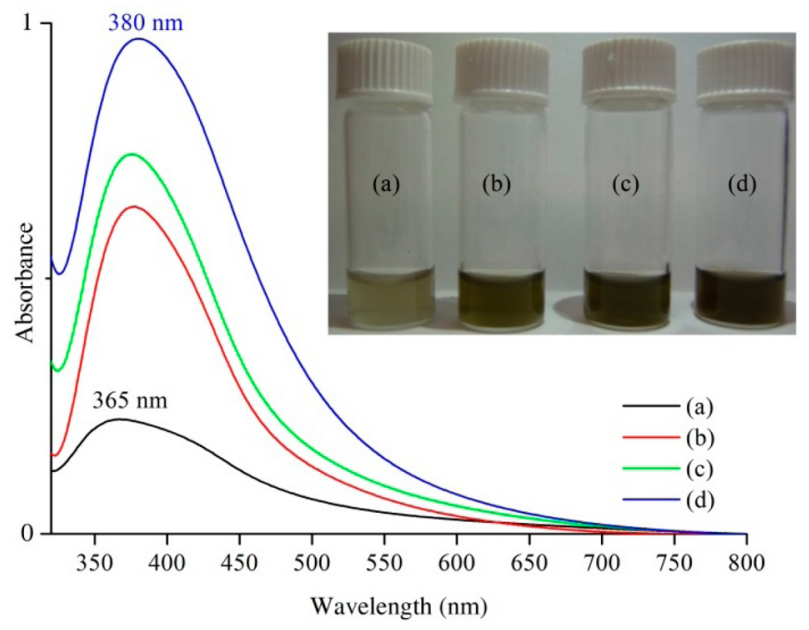

Figure 2. Evolution of the Ag plasmon band in FAU suspensions after UV treatment at $100 \%$ for (a) 5, (b) 10, (c) 15, and (d) $60 \mathrm{~s}$.

appears at $365 \mathrm{~nm}$, associated with a pale green coloration of the suspension after irradiation for $5 \mathrm{~s}$. This highlights the high efficiency of this mode of reduction, i.e., formation of Ag-NPs after irradiation for only $5 \mathrm{~s}$ instead of several hours for the chemical reduction method. ${ }^{21}$ Increasing the reaction time leads to a continuous hyperchromic shift of the plasmon band and a darkening of the zeolite suspension, indicative of the progressive formation of Ag-NPs. On the basis of the band intensity, more than $75 \%$ of the silver cations are reduced after only $15 \mathrm{~s}$. Furthermore, all silver cations are reduced after irradiation for $60 \mathrm{~s}$ with no increase in the plasmon band intensity with longer irradiation times [ 2 or 3 min (Figure S2 of the Supporting Information)]. Besides, an additional amount of reducing agent is added to the samples and then subjected to irradiation. No notable change in the plasmon band is observed under these conditions; thus, we conclude that the silver cations are fully reduced after treatment for only $1 \mathrm{~min}$. Instead, a decrease in plasmon band intensity takes place at a long irradiation time $(5 \mathrm{~min})$, probably because of a partial destruction of the zeolite nanoparticles caused by the harsh conditions used. Interestingly, no change in the absorbance wavelength is noticed; this indicates that the reaction does not occur via the formation of small Ag seeds growing into larger particles during the reaction. ${ }^{21}$ Because of the fast reaction process, Ag-NPs of a similar size are progressively produced.

The DLS study of the FAU-Ag suspensions obtained under different irradiation conditions shows a monomodal particle size distribution of particles with an average diameter of $70 \mathrm{~nm}$ (Figure 3), matching the DLS from a pure FAU zeolite

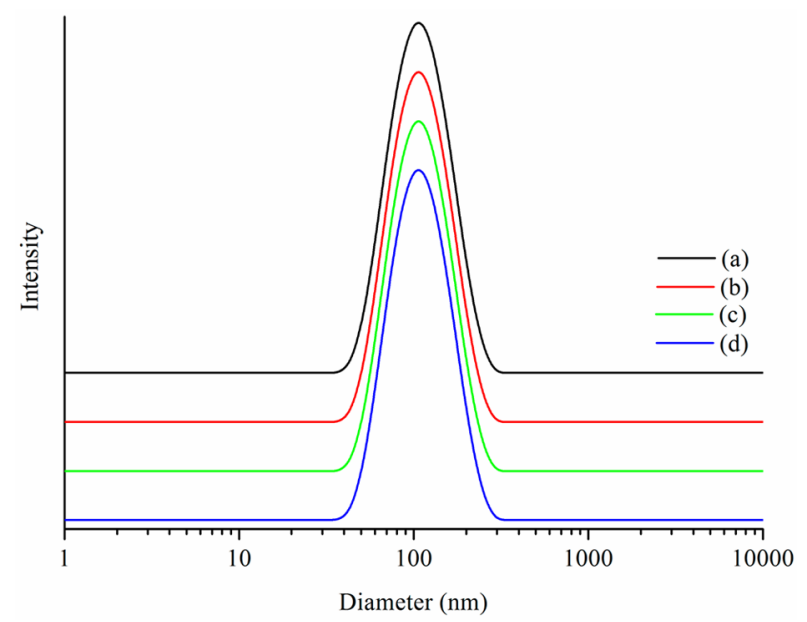

Figure 3. DLS curves of FAU-Ag suspensions after irradiation with an $I_{0}$ of $100 \%$ for (a) 5, (b) 10, (c) 15 , and (d) $60 \mathrm{~s}$.

suspension (Figure S3 of the Supporting Information). This indicates that the silver nanoparticles are completely associated with the zeolite crystals and no evidence of separate Ag-NPs is observed in the suspensions.

The DLS results are confirmed by a HRTEM study (Figure 4 and Figure S4 of the Supporting Information). The zeolite crystals contain silver nanoparticles predominantly located in the channels of FAU crystals; however, several Ag-NPs are seen on the zeolite surface. At high magnifications, the zeolite nanoparticles show a high degree of crystallinity and wellaligned crystal fringes. As previously mentioned, because of the short reaction period, the Ag nanoparticles are of similar size and shape and appear as spheres with a diameter in the range of $0.7-1.1 \mathrm{~nm}$. More than $80 \%$ of the Ag-NPs are located in the super cages $(1.1 \mathrm{~nm})$ and some in the sodalite cages $(0.7 \mathrm{~nm})$ of the FAU zeolite crystals. The entire FAU zeolite crystal containing Ag-NPs at high magnification is shown in Figure $4 \mathrm{C}$; the homogeneous distribution of Ag-NPs all over the zeolite crystal can be seen. The regular distribution of Ag-NPs in the zeolite crystals is observed at high magnifications, as shown as an inset of the enlarged selected area in Figure 4B. However, some Ag-NPs with a size of 5-6 nm can be seen on the zeolite external surface (Figure 4A).

The zeolite crystals containing Ag-NPs are further characterized by EDS-TEM (Figure 5). 

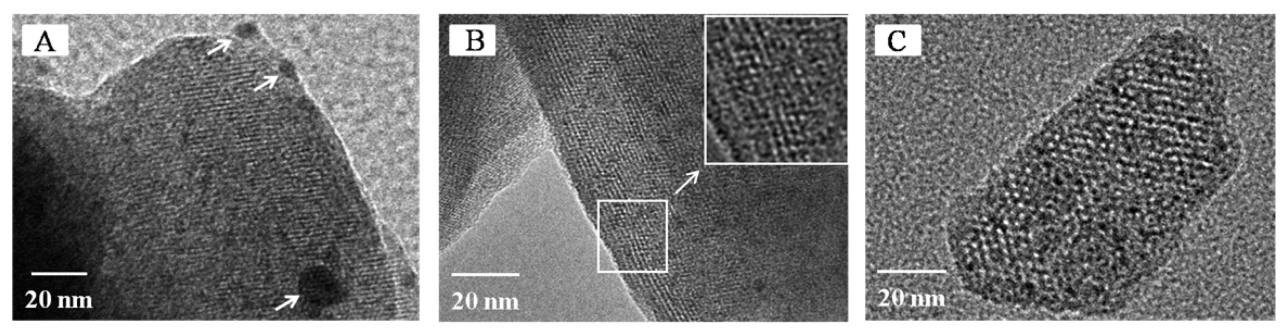

Figure 4. HRTEM images of FAU crystals containing Ag-NPs. (A) Ag-NPs within the cages and on the external surface of the zeolite crystals (arrows). (B) Ag-NPs in the zeolite cages only. The inset shows an enlarged selected area showing the silver nanoparticle in the zeolites. (C) Ag-NPs homogeneously distributed in the entire crystalline FAU zeolite particle. This sample was obtained after UV treatment at $100 \%$ for $60 \mathrm{~s}$.

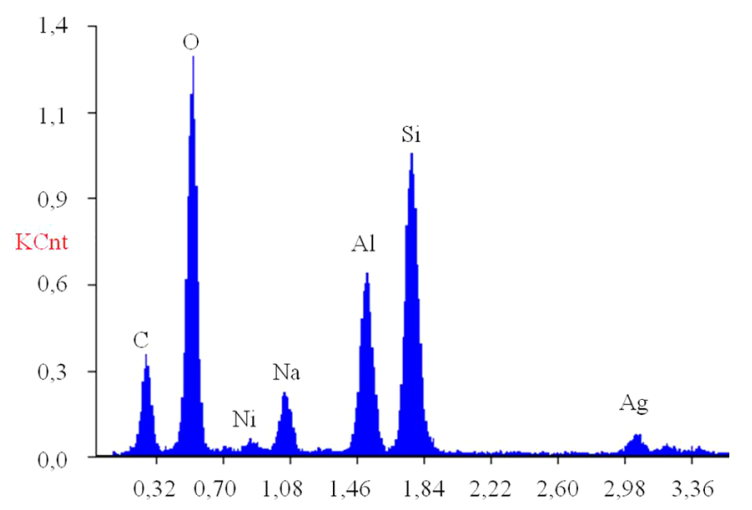

\begin{tabular}{ccc}
\hline Element & Weight \% & Atomic \% \\
\hline $\mathrm{Na}$ & 26.7 & 32.6 \\
$\mathrm{Al}$ & 28.0 & 29.2 \\
$\mathrm{Si}$ & 35.7 & 35.7 \\
$\mathrm{Ag}$ & 9.5 & 2.5 \\
\hline
\end{tabular}

Figure 5. Elemental composition of the FAU-Ag sample measured by EDX-HRTEM (C corresponds to the holey carbon film, and Ni is coming from the grid used in the TEM experiment). The inset shows the elemental analysis of the FAU-Ag sample expressed in weight and atomic percent.

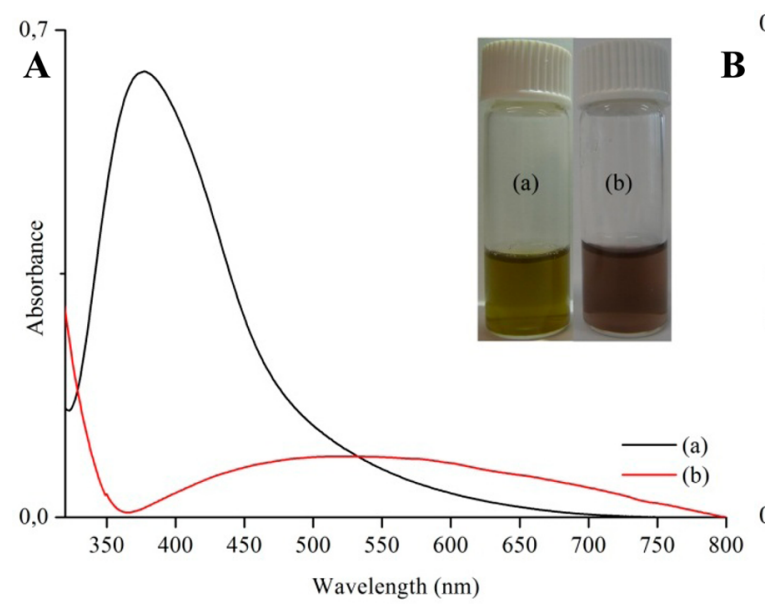

Figure 6. (A) Plasmon band of Ag-NPs in (a) a FAU-Ag suspension an plasmon band for (a) FAU-Ag and (b) pure Ag in water after $60 \mathrm{~min}$.

The zeolite main components, i.e., $\mathrm{Al}, \mathrm{Si}$, and $\mathrm{Na}$, are measured and presented in Figure 5. A Si:Al ratio of $\sim 1.2$, which is characteristic of the FAU type zeolite $(\mathrm{X})$, is measured. Additionally, the partial ion exchange of sodium for silver is evidenced. Besides, the chemical composition of the ionexchanged (FAU-Ag ${ }^{+}$) and reduced (FAU-Ag) zeolite samples is determined and presented in Table S1 of the Supporting Information. The results confirm that the silver content does not change; thus, only reduction of the silver cations occurred, leading to the formation of Ag-NPs, which is proven by UV-vis measurements.

Stability of Ag-NPs. The stability of Ag-NPs in the FAU zeolite is of prime importance. It is expected that the photoreductant has a dual action during the photoreduction



(b) pure Ag-NPs in water $\left(I_{0}=100 \%\right.$ for $\left.10 \mathrm{~s}\right)$. (B) Evolution of the process: (1) reducing the silver cations and simultaneously (2) stabilizing the Ag-NPs from further oxidation. However, we have already mentioned that, in our case, the reducing agent is fragmented during the reaction and so produces free radicals. Hence, it is not capable of ensuring the stability of the reaction products. The zeolite is therefore used as an inorganic matrix to stabilize the Ag-NPs. ${ }^{10}$ In Figure 6A, the plasmon band of AgNPs stabilized in the FAU zeolite, and a control test with the same amount of nonsupported and reduced silver in a water solution is presented. It is clearly seen that the Ag-NPs in FAU zeolite exhibit an intense and nearly symmetrical band reflecting their high degree of monodispersity, already shown by HREM. Moreover, the Ag-NPs in a water solution have a very broad and weak band extending from 365 to $800 \mathrm{~nm}$ 
(Figure 6A, black line). This band indicates the presence of AgNPs of different sizes. Hence, the first role of the zeolite crystals is to induce a homogeneous formation of Ag-NPs with a defined location within the zeolite pores, and on the zeolite surface. One hour after irradiation, the plasmon band of AgNPs supported on FAU zeolite crystals is unchanged (Figure 6B), while the corresponding band for free Ag-NPs in a water solution totally disappears. This evolution is accompanied by the discoloration of the suspension, from light brown to colorless. For the FAU-Ag suspension, no change in suspension color, plasmon band position, or intensity is observed after several months, while in the absence of zeolite, the Ag-NPs start to be oxidized after reduction for only a few minutes (Figure S5 of the Supporting Information).

Theoretical Calculations of Ag-NPs Based on the Plasmon Band. A comparative study between the experimental and calculated plasmon bands for Ag-NPs stabilized in FAU zeolites is presented in Figure 7. Simulations of Ag-NPs in

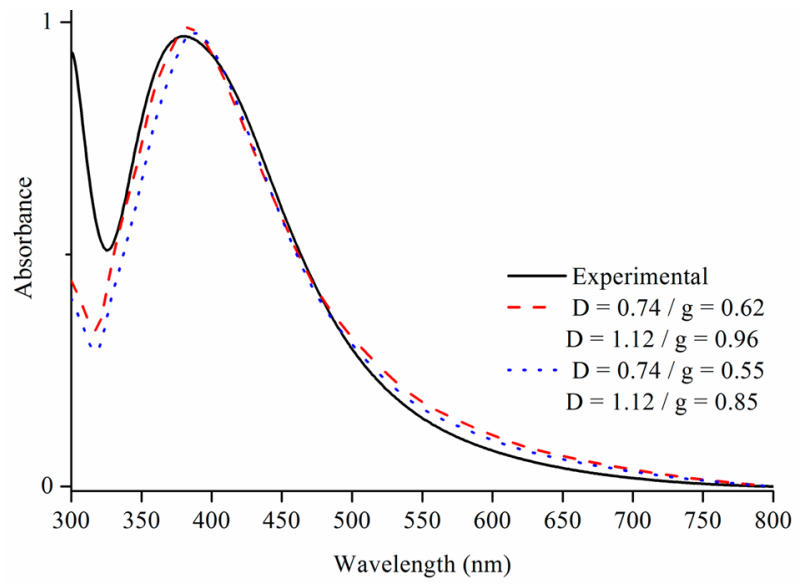

Figure 7. Experimental plasmon band of Ag-NPs in a FAU zeolite suspension irradiated with an $I$ of $100 \%$ after $60 \mathrm{~s}$ (black line) and calculated plasmon band for Ag spheres with diameters $(d)$ of 0.74 and $1.12 \mathrm{~nm}$ [parameter $g=0.62$ and $g=0.96, \mathrm{~N} m=1.28$ (red dotted line)] and calculated plasmon band for the $\mathrm{Ag}$ sphere with diameters of 0.74 and $1.12 \mathrm{~nm}$ (parameter $g=0.55$ and $g=0.85, \mathrm{~N} \_m=1.28$ ).

water are first conducted. The simulations are performed for two spheres with diameters of $0.74 \mathrm{~nm}$ (opening of the supercage) and $1.12 \mathrm{~nm}$ (diameter of the supercage, dotted blue line), using an adjustable damping parameter $(g)$, determined using a fitting algorithm. As shown in Figure 7, the $D=0.74 \mathrm{~nm}, g=0.55$ case and the $D=1.12 \mathrm{~nm}, g=0.85$ case result in similar absorption spectra. In fact, the experimental absorption spectrum can be reproduced with various values of $D$ and $g$. However, larger nanoparticle diameters would be best fit using a " $g$ " value of $>0.85$, and smaller nanoparticle diameters would be best fit with a " $g$ " value of $<0.55$. Thus, the values shown in Figure 7 present the best agreement between the diameters expected for the silver nanoparticles and the $g$ value, reported in the literature for spherical particles (i.e., $0.7 \pm 0.1$ ). The fits can be improved by changing the refractive index of the surrounding medium to a value of 1.28. Again, the experimental data can be best fit with the $D=0.74 \mathrm{~nm}, g=0.62$ case and the $D=1.12 \mathrm{~nm}, g=0.96$ case, as shown in Figure 7. Although both diameters can explain the experimental absorption spectra, additional Mie theory simulations, using adjustable Gaussian distributions of nano- particle diameters, show that the experimental results are best fit with a unique diameter. While it is difficult to ponder the relative weight of both diameters, the simulations unambiguously show that silver nanoparticles with sizes ranging between 0.74 and $1.12 \mathrm{~nm}$ are at the origin of the absorption peak at 380 $\mathrm{nm}$. The ratio of the two sizes of Ag-NPs $(D=0.735 \mathrm{~nm}$, and $D$ $=1.124 \mathrm{~nm}$ ) with different damping factors could be further investigated to determine the ratio of the opening of the super cage (sodalite cage) over the super cage.

\section{CONCLUSIONS}

A facile and rapid preparation of silver nanoparticles supported on FAU zeolite crystals (zeolite X) based on UV irradiation is reported and analyzed. A silver ion-exchanged FAU zeolite suspension is irradiated with a $\mathrm{Xe}-\mathrm{Hg}$ lamp (200 W) in the presence of a photoactive reducing agent (2-hydroxy-2methylpropiophenone). Silver nanoparticles are prepared after a very short irradiation time (5-60 s), and a faster reduction is achieved after optimizing the process conditions at higher irradiation intensities. The optimal conditions for fully reducing the silver cations are a lamp intensity $\left(I_{0}\right)$ of $100 \%$ for $60 \mathrm{~s}$.

The size, morphology, and distribution of Ag-NPs in the FAU zeolite crystals are characterized by HRTEM. The Ag-NPs are located predominantly inside the micropores and to a lesser extent on the external surface of the FAU zeolite crystals. The Ag-NPs have an almost spheroidal shape with a diameter of 0.74-1.124 nm, which corresponds to the size of the cage opening (sodalite cage) and super cage of FAU type zeolite. This is further confirmed by a theoretical modeling of the plasmon band. The Ag-NPs in the FAU zeolite crystals without stabilizers or capping agents exhibit high stability and, after several months, do not change, again because of the stabilizing role of the zeolite nanocrystals.

\section{ASSOCIATED CONTENT}

\section{S Supporting Information}

UV-vis spectra of the FAU- $\mathrm{Ag}^{+}$suspension without and with a photoreducing agent (2-hydroxy-2-methylpropiophenone) at time zero (Figure S1), evolution of the Ag plasmon band in the FAU-Ag suspension after UV treatment at an $I_{0}$ of $100 \%$ for 1 , 2 , and $3 \mathrm{~min}$ (Figure S2), DLS curve of the FAU zeolite suspension with a solid content of 1 wt \% (Figure S3), TEM picture of the FAU-Ag sample $(M=100 \mathrm{~nm})$ (Figure S4), evolution of the plasmon band of Ag-NPs with time after irradiation of $\mathrm{AgNO}_{3}$ in water (Figure S5), modeling of $\mathrm{Ag} \mathrm{NPs}$ based on the UV-vis spectra, and elemental analysis of the FAU-Ag sample before and after reduction expressed in weight and atomic percent (Table S1). This material is available free of charge via the Internet at http://pubs.acs.org.

\section{AUTHOR INFORMATION}

\section{Corresponding Author}

*E-mail: svetlana.mintova@ensicaen.fr.

\section{Notes}

The authors declare no competing financial interest.

\section{ACKNOWLEDGMENTS}

The financial support from the SOLAIRE Emergent Region Project of Lower Normandy and the MEET, INTERREG EC project is acknowledged. We acknowledge Hussein Awala for the preparation of FAU zeolite crystals. 


\section{REFERENCES}

(1) Flores-Lopez, N. S.; Castro-Rosas, J.; Ramirez-Bon, R.; MendozaCordova, A.; Larios-Rodriguez, E.; Flores-Acosta, M. Synthesis and properties of crystalline silver nanoparticles supported in natural zeolite chabazite. J. Mol. Struct. 2012, 1028, 110-115.

(2) Tung, N. H.; Chikae, M.; Ukita, Y.; Viet, P. H.; Takamura, Y. Sensing technique of silver nanoparticles as labels for immunoassay using liquid electrode plasma atomic emission spectrometry. Anal. Chem. 2012, 84, 1210-1213.

(3) Liu, F.; Rao, B. S.; Nunzi, J.-M. A dye functionalized silver-silica core-shell nanoparticle organic light emitting diode. Org. Electron. 2011, 12, 1279-1284.

(4) Noh, H. S.; Cho, E. H.; Kim, H. M.; Han, Y. D.; Joo, J. Organic solar cells using plasmonics of Ag nanoprisms. Org. Electron. 2013, 14, 278-285.

(5) Kulkarni, A. P.; Noone, K. M.; Munechika, K.; Guyer, S. R; Ginger, D. S. Plasmon-enhanced charge carrier generation in organic photovoltaic films using silver nanoprisms. Nano Lett. 2010, 10, 15011505.

(6) Atwater, H. A.; Polman, A. Plasmonics for improved photovoltaic devices. Nat. Mater. 2010, 3, 205-213.

(7) Cuong, N. H.; Nguyen, H. M. T.; Nguyen, M. T. Theoretical modeling of optical properties of $\mathrm{Ag}^{8}$ and $\mathrm{Ag}^{14}$ silver clusters embedded in an LTA sodalite zeolite cavity. Phys. Chem. Chem. Phys. 2013, 15, 15404-15415.

(8) Miyanaga, T.; Suzuki, Y.; Matsumoto, N.; Narita, S.; Ainai, T.; Hoshino, H. Formation of Ag clusters in zeolite $\mathrm{X}$ studied by in situ EXAFS and infrared spectroscopy. Microporous Mesoporous Mater. 2013, 168, 213-220.

(9) Lee, J.-H.; Hwang, J.-H.; Nam, J.-M. DNA-tailored plasmonic nanoparticles for biosensing applications. WIREs Nanomedicine and Nanobiotechnology 2013, 5, 96-109.

(10) Zaarour, M.; Dong, B.; Naydenova, I.; Retoux, R.; Mintova, S. Progress in zeolite synthesis promotes advanced applications. Microporous Mesoporous Mater. 2014, 189, 11-21.

(11) Devaux, A.; Calzaferri, G.; Miletto, I.; Cao, P.; Belser, P.; Bruhwiler, D.; Khorev, O.; Robert Haner, R.; Kunzmann, A. Selfabsorption and luminescence quantum yields of dye-zeolite $\mathrm{L}$ composites. J. Phys. Chem. C 2013, 117, 23034-23047.

(12) Yang, M.; Fujino, T. Silver nanoparticles on zeolite surface for laser desorption/ionization mass spectrometry of low molecular weight compounds. Chem. Phys. Lett. 2013, 576, 61-64.

(13) Guerra, R.; Lima, E.; Viniegra, M.; Guzman, A.; Lara, V. Growth of Escherichia coli and Salmonella typhi inhibited by fractal silver nanoparticles supported on zeolites. Microporous Mesoporous Mater. 2012, 147, 267-273.

(14) Liu, Y.; Zhu, Z.; Liu, G.; Xu, Z.; Kuznicki, S. M.; Zhang, H. A novel method to improve crystallinity of supported nanoparticles using low melting point metals. J. Phys. Chem. C 2011, 115, 14591-14597.

(15) Cavicchioli, M.; Varanda, L. C.; Massabni, A. C.; Melnikov, P. Silver nanoparticles synthesized by thermal reduction of a silver(I)aspartame complex in inert atmosphere. Mater. Lett. 2005, 28, 35853589.

(16) Aswathy, B.; Avadhani, G. S.; Sumithra, I. S.; Suji, S.; Sony, G. Microwave assisted synthesis and UV-Vis spectroscopic studies of silver nanoparticles synthesized using vanillin as a reducing agent. $J$. Mol. Liq. 2011, 159, 165-169.

(17) Wani, I. A.; Ganguly, A.; Ahmed, J.; Ahmad, T. Silver nanoparticles: Ultrasonic wave assisted synthesis, optical characterization and surface area studies. Mater. Lett. 2011, 65, 520-522.

(18) Rycenga, M.; Cobley, C. M.; Zeng, J.; Li, W.; Moran, C. H.; Zhang, Q.; Qin, D.; Xia, Y. Controlling the synthesis and assembly of silver nanostructures for plasmonic applications. Chem. Rev. 2011, 111, 3669-3712.

(19) Jia, H.; Zeng, J.; Song, W.; An, J.; Zhao, B. Preparation of silver nanoparticles by photo-reduction for surface-enhanced Raman scattering. Thin Solid Films 2006, 2, 281-287.

(20) Tsuji, T.; Okazaki, Y.; Tsuji, M. Photo-induced morphological conversions of silver nanoparticles prepared using laser ablation in water-enhanced morphological conversions using halogen etching. J. Photochem. Photobiol., A 2008, 194, 247-253.

(21) Kshirsagar, P.; Sangaru, S. S.; Malvindi, M. A.; Martiradonna, L.; Cingolani, R.; Pomp, P. P. Synthesis of highly stable silver nanoparticles by photoreduction and their size fractionation by phase transfer method. Colloids Surf., A 2011, 392, 264-270.

(22) Bohren, C. F.; Huffman, D. R. Absorption and Scattering of Light by Small Particles; John Wiley \& Sons: New York, 1998.

(23) International Association for the Properties of Water and Steam. Release on Refractive Index of Ordinary Water Substance as a Function of Wavelength, Temperature and Pressure, 1997.

(24) Johnson, P. B.; Christy, R. W. Optical Constants of the Noble Metals. Phys. Rev. 1972, B6, 4370-4379.

(25) Kreibig, U. Electronic properties of small silver particles: The optical constants and their temperature dependence. J. Phys. F: Met. Phys. 1974, 4, 999-1014.

(26) Lermé, J.; Baida, H.; Bonnet, C.; Broyer, M.; Cottancin, E.; Crut, A.; Maioli, P.; Del Fatti, N.; Valle, F.; Pellarin, M. Size Dependence of the Surface Plasmon Resonance Damping in Metal Nanospheres. J. Phys. Chem. Lett. 2010, 1, 2922-2928.

(27) Zeman, E. J.; Schatz, G. C. An accurate electromagnetic theory study of surface enhancement factors for silver, gold, copper, lithium, sodium, aluminum, gallium, indium, zinc, and cadmium. J. Phys. Chem. 1987, 91, 634-643.

(28) Andrzejewska, E. Photopolymerization kinetics of multifunctional monomers. Prog. Polym. Sci. 2001, 26, 605-665. 